\title{
DMPO-OH Radical Formation from 5,5-Dimethyl-1-pyrroline $N$-Oxide (DMPO) in Hot Water
}

\author{
Tomoko ShoJI, ${ }^{* 1}$ Linxiang LI, ${ }^{* 1}$ Yoshihiro Abe, ${ }^{* 1 \dagger}$ Masahiro OGata, ${ }^{* 2}$ Yoshihisa IshImoto, ${ }^{* 1}$ \\ Ryoko Gonda, ${ }^{* 1}$ Tadahiko MAShIno, ${ }^{* 1}$ Masataka Mochizuki, ${ }^{* 1}$ Michihisa Uemoto, ${ }^{* 3}$ and \\ Naoki MIYATA*4 \\ *1 Kyoritsu University of Pharmacy, 1-5-30 Shibakoen, Minato, Tokyo 105-8512, Japan \\ *2 Faculty of Pharmaceutical Sciences, Aomori University, 2-3-1 Koubata, Aomori 030-0943, Japan \\ *3 Tokyo Metropolitan Industrial Technology Research Institute, 3-13-10 Nishigaoka, Itabashi, \\ Tokyo 115-8586, Japan \\ *4 Faculty of Pharmaceutical Sciences, Nagoya City University, 3-1 Tanabe-dori, Mizuho, \\ Nagoya 467-8603, Japan
}

\begin{abstract}
When an aqueous solution of 5,5-dimethyl-1-pyrroline $N$-oxide (DMPO) was heated at $70^{\circ} \mathrm{C}$ for 30 min, formation of DMPO-OH was observed by ESR. This DMPO-OH radical formation was suppressed under an argon atmosphere. When water was replaced with ultra-pure water for ICP-MS experiments, DMPO-OH radical formation was also diminished. Under an argon atmosphere in ultra-pure water, the intensity of the DMPO-OH signal decreased to about $1 / 20$ of that observed under aerobic conditions with regular purified water. The addition of hydroxyl radical scavengers such as mannitol did not affect the formation of DMPO-OH, but the signal turned faint in the presence of EDTA. We suggest that DMPO reacted with dissolved oxygen to form DMPO-OH.
\end{abstract}

(Received August 7, 2006; Accepted November 9, 2006; Published February 10, 2007)

\section{Introduction}

The spin-trapping method with 5,5-dimethyl-1-pyrroline $\mathrm{N}$ oxide (DMPO) has been widely accepted as an assay method to measure hydroxyl radical formation, and to detect the hydroxyl radical scavenging activity of a compound. Although unstability of DMPO is claimed because it turns yellow with time even at $-20^{\circ} \mathrm{C}$ in a sealed tube under vacuum, ${ }^{1}$ the basic chemistry of DMPO and DMPO-OH has not been well understood.

In a previous work, ${ }^{2}$ we noticed very weak DMPO-OH signals in the baseline of a negative control spectrum which was obtained after standing DMPO solution at $37^{\circ} \mathrm{C}$ for $24 \mathrm{~h}$. We suspected that in a DMPO aqueous solution at a higher concentration and at a higher temperature, an appropriate amount of DMPO-OH might generate. And we considered that this reaction should provide a practical preparation method to obtain DMPO-OH radical as a simple aqueous solution which contains no other materials except unreacted DMPO. Also this DMPO-OH aqueous solution may be utilized in the investigation of the chemical or physicochemical properties of DMPO-OH radicals.

When DMPO was dissolved in purified water and the solution was heated at $70^{\circ} \mathrm{C}$ for $30 \mathrm{~min}$, a sufficient amount of DMPO$\mathrm{OH}$ radical formation was observed by ESR (Fig. 1). Under an argon atmosphere, DMPO-OH formation was minimized to $1 / 4$, and this slightly increased under a dioxygen atmosphere. This indicates that dioxygen participates in the reaction. Previously, Makino et al. observed DMPO-OH formation in an aqueous mixture of DMPO and $1 \mathrm{mM} \mathrm{FeCl}{ }_{3}$. Based on this observation

† To whom correspondence should be addressed. of the formation of iron chelate with DMPO at $77 \mathrm{~K}$, and DMPO-OCH $\mathrm{H}_{3}$ formation in the presence of $\mathrm{CH}_{3} \mathrm{OH}$, researchers elucidated that nucleophilic attachment of water to DMPO should occur in the presence of $\mathrm{Fe}^{3+}$ ion. ${ }^{3}$ To study the effect of a small number of metal ions in purified water, we replaced the purified water with ultra-pure water for ICP-MS measurements; the latter should contain iron of no more than $1 \mathrm{ppb}$. In ultrapure water, and under an argon atmosphere, DMPO-OH signal was still observed after heating, although its intensity decreased to about $1 / 20$. In this communication, we describe DMPO-OH radical formation in hot water, in which dissolved dioxygen participates in the reaction and metal ions such as $\mathrm{Fe}^{3+}$ might catalyze DMPO-OH formation.

\section{Experimental}

\section{Chemicals}

5,5-Dimethyl-1-pyrroline $N$-oxide (DMPO) was obtained from

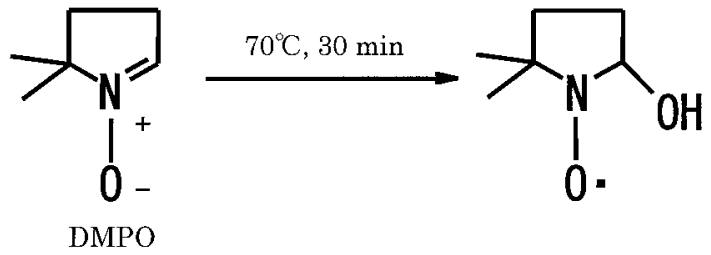

Fig. 1 DMPO-OH radical formed in a heated aqueous solution of DMPO. 


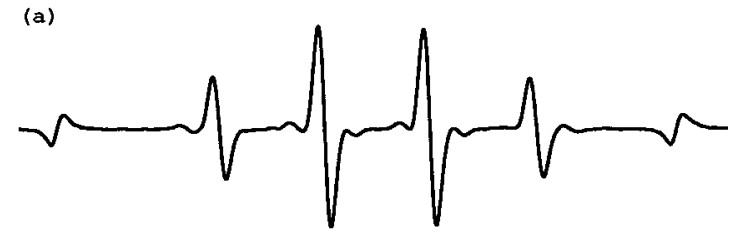

(b)

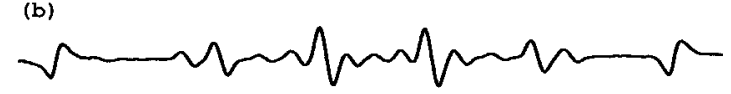

(c)
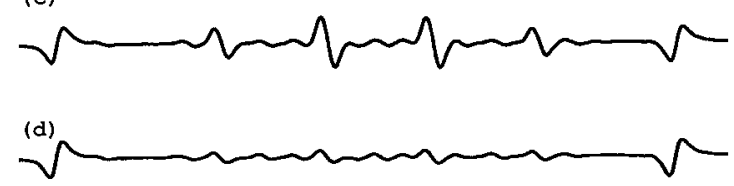

10G

Fig. 2 The ESR spectrum of DMPO-OH radical observed in an aqueous solution of DMPO after heating at $70^{\circ} \mathrm{C}$ for $30 \mathrm{~min}$ (a). Four strong signals in the mid-section are of the DMPO-OH radical $\left(A_{\mathrm{H}}\right.$ $1.50 \mathrm{mT}, A_{\mathrm{N}} 1.50 \mathrm{mT}$ ), and two side signals are of Mn used as an external reference. The ESR spectrum of DMPO aqueous solution heated under an argon atmosphere (b); under aerobic conditions in ultra-pure water (c); under an argon atmosphere in ultra-pure water (d).

Labotec Co. (Tokyo, Japan), and used without further purification. The water used in this study was purified by ion-exchange resin (18.2 M $\Omega$ ). Ultra-pure water for ICP-MS was obtained by two successive distillations of purified water with an all-quartz apparatus, in which $\mathrm{Fe}^{3+}$ ion was not detected (at most $1 \mathrm{ppb}$ ). In experiments under an argon atmosphere, water was deaerated under reduced pressure using an aspirator, and then stored under argon by attaching an argon balloon.

\section{ESR measurement}

An electron spin resonance spectrometer, JES-RE1X (JEOL, Tokyo, Japan), and a JEOL flat quartz cell were used. The conditions were: field, $336 \pm 5 \mathrm{mT}$ width; power, $4 \mathrm{~mW}$; field modulation, $0.200 \mathrm{mT}$; time constant, 0.1; and amplitude, 500. A manganese signal was used for the external standard. Pyrex glassware was washed with $1 \mathrm{M}$ nitric acid by sonication, rinsed well with purified water, and air dried.

\section{DMPO-OH radical formation in hot water}

Neat DMPO was dissolved in purified water to make a $2.5 \%$ aqueous solution. In cases of $\mathrm{pH}$ controlled experiments, $2.5 \%$ DMPO solution in an acetate buffer or a phosphate buffer was used. A 2-mL volume of sample was heated in a test tube for $30 \mathrm{~min}$ in a water bath at $70^{\circ} \mathrm{C}$. Under an argon atmosphere or a dioxygen atmosphere, $10 \mathrm{~mL}$ of $2.5 \%$ DMPO aqueous solution was introduced into a $25 \mathrm{~mL}$ round-bottomed flask, and a threeway stopcock with an argon (or dioxygen) balloon was attached. The solution was deaerated under reduced pressure (aspirator), and argon (or dioxygen) was introduced from the balloon. After heating at $70^{\circ} \mathrm{C}$ for $30 \mathrm{~min}$, an aliquot was removed and was measured by ESR just 1 min after the tube was taken out of the water bath.

\section{Results and Discussion}

After heating the aqueous solution of DMPO at $70^{\circ} \mathrm{C}$ for 30

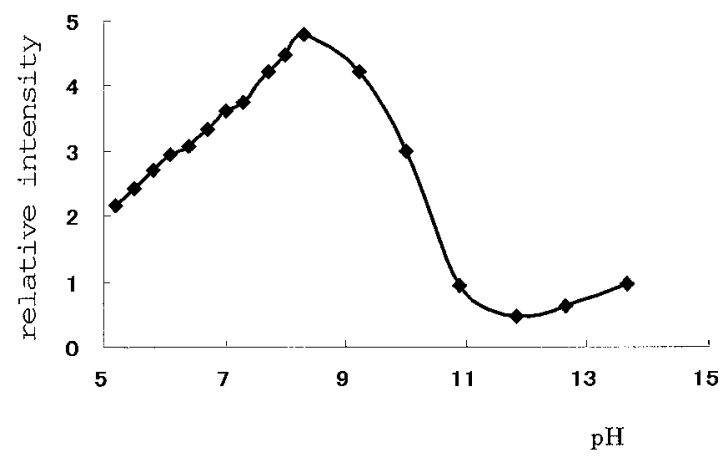

Fig. 3 Effect of $\mathrm{pH}$ on the relative intensity of DMPO-OH vs. Mn (external reference), which is the highest at around $\mathrm{pH} 8.5$.

min, we observed typical ESR signals of DMPO-OH radical with an intensity ratio of $1: 2: 2: 1\left(A_{\mathrm{H}} 1.50 \mathrm{mT}, A_{\mathrm{N}} 1.50 \mathrm{mT}\right.$; Ref. 4, $A_{\mathrm{H}} 1.53 \mathrm{mT}, A_{\mathrm{N}} 1.53 \mathrm{mT}$ ) (Figs. 1 and 2).

It was reported that $\mathrm{Fe}^{3+}$ ions catalyze the addition of water to DMPO to produce DMPO-OH radical. ${ }^{3}$ In Fig. 2, however, its formation decreased under Ar atmosphere (Figs. 2(b) and (d)), also in ultra-pure water under aerobic conditions (Fig. 2(c)). In all spectra in Fig. 2, unknown weak signals can be seen. DMPO-OH radical formation by heating was not suppressed by the addition of a hydroxyl radical scavenger, mannitol (1-100 $\mathrm{mM})$ or DMSO $(1-100 \mathrm{mM})$. This indicates that DMPO-OH radical should not be formed via hydroxyl radical attachment to DMPO. However, DMPO-OH signal intensity was decreased to about $1 / 6$ in the presence of $1 \mathrm{mM}$ of EDTA. Thus, it was suspected that dissolved dioxygen, which is a biradical molecule, should be added directly to DMPO, where catalytic participation of metal ions might exist (Eq. (1)). Biradical might produce a DMPO-OOH radical, and this would develop into a DMPO-OH radical quickly at higher temperatures.

DMPO-OH signals were also observed in acetate buffer $(\mathrm{pH}$ 5.0-6.8), phosphate buffer ( $\mathrm{pH} 7.0-10.0$ ) or disodium phosphate solution of which the $\mathrm{pH}$ was adjusted with sodium hydroxide solution ( $\mathrm{pH}$ 13.0) (Fig. 3). The half-lives of DMPO$\mathrm{OH}^{5}$ or DMPO-OOH ${ }^{6,7}$ at basic $\mathrm{pH}$ are much shorter than those at neutral or acidic conditions, and the decrease of the intensity of DMPO-OH signal can be considered as the unstability of these radical species. The optimum $\mathrm{pH}$ was found to be around $\mathrm{pH} 8.5$, thus, the catalytic action of $\mathrm{H}^{+}$may not be plausible.

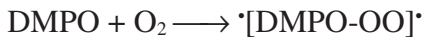

$$
\begin{aligned}
& \cdot\left[\mathrm{DMPO}-\mathrm{O}_{2}\right]^{\cdot}+\mathrm{H}_{2} \mathrm{O} \longrightarrow[\text { DMPO-OOH }]^{\cdot}+{ }^{\circ} \mathrm{OH} \\
& {[\mathrm{DMPO}-\mathrm{OOH}]^{\cdot} \longrightarrow[\mathrm{DMPO}-\mathrm{OH}]^{\cdot}}
\end{aligned}
$$

Even at low concentrations, transition metal ions such as $\mathrm{Fe}^{3+}$ may catalyze the first step (Eq. (1)), or catalyze the reaction of $\left[\mathrm{DMPO}^{-\mathrm{O}_{2}}\right]_{\text {biradical with water to [DMPO-OOH }}{ }^{*}$ (Eq. (2)), although we could not detect [DMPO-OOH] signals nor $\mathrm{OH}$ radical formation by the addition of DMSO. DMPO-OOH radical is known to be labile so as to decompose to DMPO-OH with a half-time of $50 \mathrm{~s}$ at $\mathrm{pH} 7^{6,7}$ (Eq. (3)), and the decomposition mechanisms of DMPO-OOH adducts to DMPO$\mathrm{OH}$ are discussed by Villamena et al. ${ }^{8}$

The formation of DMPO-OH with time was traced at $70^{\circ} \mathrm{C}$ (Fig. 4). It was found that the reaction reached almost steady state after $60 \mathrm{~min}$. After dioxygen in the reaction mixture is consumed, the supply of dioxygen to the reaction solution 


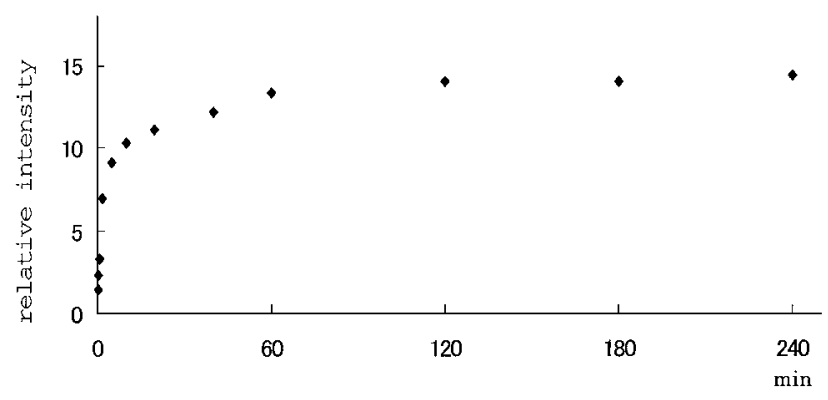

Fig. 4 The relative intensity of DMPO-OH vs. Mn (external reference) with time. The ESR signal of DMPO-OH radical in a 2.5\% DMPO solution adjusted at $\mathrm{pH} 7.4$ using a phosphate buffer, was recorded just $1 \mathrm{~min}$ after taking out the reaction tube which was heated at $70^{\circ} \mathrm{C}$ and then cooled at $0^{\circ} \mathrm{C}$ for $15 \mathrm{~s}$ in a water-bath.

DMPO-OH formation could be neglected.

The mechanism of DMPO-OH radical formation remains uncertain; however, we report here the observed evidence that DMPO should react with dioxygen in water to generate DMPO$\mathrm{OH}$ radical under aerobic conditions at $70^{\circ} \mathrm{C}$.

\section{Conclusions}

In conclusion, DMPO-OH radical was detected after heating a DMPO aqueous solution at $70^{\circ} \mathrm{C}$ for $30 \mathrm{~min}$. In the presence of dioxygen, DMPO-OH production was enhanced. The catalytic participation of metal ions was also suspected, because decreased formation of DMPO-OH was observed when regular purified water was replaced with ultra-pure water.

\section{References}

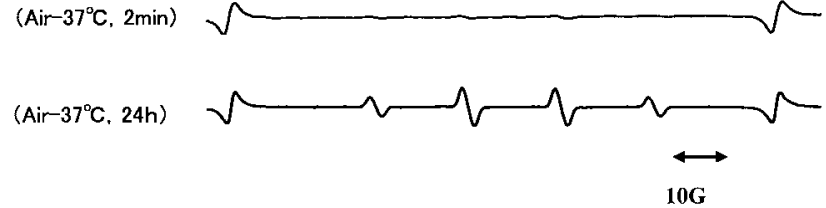

Fig. 5 ESR signal of DMPO-OH radical observed in an aqueous solution of DMPO (dissolved in milliQ water) under aerobic conditions.

should be the rate-determining step.

DMPO-OH formation in an aqueous solution of DMPO was also observed at $37^{\circ} \mathrm{C}$ after $24 \mathrm{~h}$, indicating that radical trapping experiments in an intact animal using DMPO over $20 \mathrm{~h}$ may provide misleading results (Fig. 5). However, in the standard experimental procedure, spin-trapping experiments should be carried out at room temperature or lower, and over a short time,
1. "Merck Index", 20th ed., 2001, Merck \& Co. Inc., NJ, USA, 597.

2. M. Ogata, D. Kaneya, K. Shin-ya, L. Li, Y. Abe, H. Kato, S. Seki, Y. Seki, R. Gonda, S. Urano, and T. Endo, Chem. Pharm. Bull., 2005, 93, 1167.

3. K. Makino, A. Hagi, H. Ide, and A. Murakami, Can. J. Chem., 1992, 70, 2818.

4. J. R. Harbour, V. Chow, and J. R. Bolton, Can. J. Chem., 1974, 52, 3549.

5. P. R. Marrriot, M. J. Parkins, and D. Griller, Can. J. Chem., 1980, 58, 803 .

6. G. R. Buettner and L. M. Oberley, Biochem. Biophys. Res. Commun., 1978, 83, 69 .

7. B. Tuccio, R. Lauricella, J. C. Bouteiller, and P. Tordo, J. Chem. Soc. Perkin Trans., 1995, 2, 295.

8. F. A. Villamena, J. K. Merle, C. M. Hadad, and J. L. Zweier, J. Phys. Chem. A, 2005, 109, 6089. 